\title{
Implementation of a standardized protocol to manage elderly patients with low energy pelvic fractures: can service improvement be expected?
}

\author{
Nikolaos K. Kanakaris ${ }^{1,2,3}$ - Tess Greven ${ }^{3,4}$ Robert M. West ${ }^{5}$ Arie B. Van Vugt ${ }^{6}$. \\ Peter V. Giannoudis ${ }^{3,7}$
}

Received: 16 March 2017 / Accepted: 30 June 2017 / Published online: 21 July 2017

(C) The Author(s) 2017. This article is an open access publication

\begin{abstract}
Purpose The incidence of low energy pelvic fractures (FPFs) in the elderly is increasing. Comorbidities, decreased bonequality, problematic fracture fixation and poor compliance represent some of their specific difficulties. In the absence of uniform management, a standard operating procedure (SOP) was introduced to our unit, aiming to improve the quality of services provided to these patients.

Methods A cohort study was contacted to test the impact of (1) using a specific clinical algorithm and (2) using different antiosteoporotic drugs. Multivariate regression analysis was used to determine prognostic factors. Study endpoints were the time-to-healing, length-of-stay, return to pre-injury mobility, union status, mortality and complications.

Results A total of 132 elderly patients ( $\geq 65$ years) admitted during the period 2012-2014 with FPFs were enrolled. Highenergy fractures, acetabular fractures, associated trauma affecting mobility, pathological pelvic lesions and operated FPFs were used as exclusion criteria. The majority of included patients were females $(108 / 132 ; 81.8 \%)$, and the mean age
\end{abstract}

Nikolaos K. Kanakaris

n.kanakaris@nhs.net; nikolaoskanakaris@yahoo.co.uk

Tess Greven

tessgreven89@gmail.com

Robert M. West

R.M.West@leeds.ac.uk

Arie B. Van Vugt

abvanvugt@planet.nl

Peter V. Giannoudis

pgiannoudi@aol.com

1 Clinical Lead Major Trauma Centre, Academic Department of Trauma \& Orthopaedics, Leeds Teaching Hospitals, Leeds, UK was 85.8 years (range 67-108). Use of antiosteoporotics was associated with a shorter time of healing $(p=0.036)$. Patients treated according to the algorithm showed a significant protection against malunion $(p<0.001)$. Also, adherence to the algorithm allowed more patients to return to their pre-injury mobility status $(p=0.039)$.

Conclusions The use of antiosteoporotic medication in elderly patients with fragility pelvic fractures was associated with faster healing, whilst the adherence to a structured clinical pathway led to less malunions and non-unions and return to pre-injury mobility state.

Keywords Elderly $\cdot$ Pelvic fracture $\cdot$ Osteoporosis $\cdot$ Standard operating procedure $\cdot$ Fragility fractures

\section{Introduction}

Pelvic fractures in the elderly are known to have distinct differences compared to those in young adults. In the latter, these

2 Academic Department of Trauma and Orthopaedics, Leeds General Infirmary, Clarendon Wing, Level A, Leeds, West Yorkshire LS13EX, UK

Academic Department of Trauma \& Orthopaedics, School of Medicine, University of Leeds, Leeds, UK

4 Medisch Spectrum Twente, Enschede, The Netherlands

5 Department of Biostatistics, University of Leeds, Leeds, UK

6 Department of Trauma Surgery, Medisch Spectrum Twente, Enschede, The Netherlands

7 NIHR Leeds Biomedical Research Unit, Chapel Allerton Hospital, Leeds, UK 
injuries are usually the result of high-energy trauma [1], whereas in the former group these are mostly low-energy injuries, secondary to falls, or repeated stresses to osteopenic/ osteoporotic bone, the so-called "fragility pelvic fractures" (FPFs) [2]. Noteworthy, in terms of hospital admissions FPFs are as common as the high-energy pelvic fractures [3], and when compared to fragility hip fractures the ratio is equal to 1:5. The incidence has increased in recent decades, and the expectations are that this growth will continue mirroring the ageing of the population, and the increasing awareness of their significance [4].

Fragility pelvic fractures are thought to be a relatively simple condition. They have been managed symptomatically for years, often without complete diagnostic investigations and limited follow up [5]. However, they can result in significant morbidity and high costs, including prolonged hospitalization, decrease in mobility, loss of autonomy, and increase in institutionalization [6]. The survival of patients with a FPF is significantly worse than that of the general population of the same age, with an even higher mortality than that of patients with a hip fracture. Interestingly, risk factors and characteristics of FPFs are similar to that of hip fractures: age $\geq 65$ years, female predominance, tendency for falls, and presence of decreased bone density [7].

While, significant effort and resources have been invested globally to optimize the management of fragility hip fractures (FHFs), FPFs have not received similar attention, and still, national or international guidelines are not available.

In our institution, it has been our observation that this cohort of patients has been managed erratically over the years between different disciplines or even individual clinicians in the absence of a uniform approach. To address this issue and to implement a service improvement, we introduced a clinical algorithm (SOPstandard operating procedure) for elderly patients with FPFs (Figs. 1 and 2). According to this SOP and since 2011, every patient admitted with a FPF underwent a CT-scan at the early stages of admission. Based on an accurate diagnosis of the disruption of the pelvic ring, each patient, who required nonoperative treatment, was managed according to a structured mobilization plan. Patients who met the indications for operative treatment followed a similar mobilization pathway postoperatively, aiming to protect the fracture site from secondary displacement and implant failure.

In this study, we tested the hypothesis that elderly patients with FPFs managed non-operatively according to the SOP would have improved functional and radiological outcome compared to patients treated in our unit before the implementation of the SOP. The patients prior to introduction of the SOP were managed with minimal diagnostic imaging, mobilization as pain allowed, and referral to the orthopaedic trauma department when a complication occurred. Moreover, we attempted to test the additional hypothesis that the use of antiosteoporotic agents would have a positive effect on the outcome of these patients.

\section{Methods}

In accordance with the NHS act of 2006, section 251, the study was carried out with the required institutional board approval under reference number 10444 . We performed a cohort study over a three-year period: from 1 January 2012 till 31 December 2014. Randomization was not applicable, whilst blinding was applied during the radiological assessment. Group A consisted of patients who were managed prior to the SOP implementation (1 January 2012 to 31 December 2012). Group B (study group) consisted of patients who were clearly managed with the SOP and their course of treatment was prospectively documented (1 January 2013 to 31 December 2014).

Inclusion criteria were elderly patients (age $\geq 65$ years at the time of injury) with a low-energy pelvic fracture that were admitted to our institution. Patients with incomplete imaging, high-energy trauma, pathological pelvic fractures, pure acetabular fractures, or co-existing other injuries that could interfere with mobilization, as well as operatively-treated FPFs were excluded.

Data on patient characteristics were collected based on other publications $[2,8]$ and clinical endpoints. These included: patient demographics, injury characteristics, hospitalization details, comorbidities, imaging results (recent DEXA scans [ \pm 1 year], X-rays, CT/MRI-scans), adherence or not to the departmental SOP (Figs. 1 and 2), pre- and post-injury ambulation, residence status, out-patient follow-up at six weeks, three, six and 12 months, complications and mortality.

Anatomical descriptions were based on multiple classification systems, i.e. Tile [9], Burgess et a. [10], Denis et al. [11], Pohlemann et al. [12] and Starr et al. [13]. The radiological assessment of the initial fracture type, as well as the progress of radiological healing was carried out by two independent raters, blinded to the other study parameters. Diagnosis and classification of the different pelvic injuries was based on plain X-ray imaging (AP-pelvis and inlet-outlet images) and/ or subsequent CT/MRI-scans performed at the early stages of diagnosis (within the first week in group B and within three weeks in group A).

Sacral lesions were graded according to the Hannover system [12] and were divided into two groups: (1) a more stable group (Hannover type 0-1a-1b), and (2) less stable group (Hannover 2a-2b-3a-3b-3c-4). Comorbidities were scored using the Charlson comorbidity index as a quantitative surrogate marker of pre-existing medical conditions. Antiosteoporotic therapy was defined as the administration of either calcium/vitamin $\mathrm{D}$, and/or bisphosphonates, strontium ranelate, or teriparatide over the period spanning from before the date of the accident to the first six weeks post-injury. Taking into account that any anti-osteoporotic therapy needs a certain period of time since its initiation to have a measurable effect on fracture healing [14], we compared patients that 
Fig. 1 The algorithm of non-operative management (FPF-SOP—standard operating procedure) of low energy fractures of the pelvic ring that was tested in this study. $A P$ anteroposterior $\mathrm{X}$-ray, $C D U$ clinical decision unit, $C T$ computed tomography, $D / W$ discuss with, FABER test flexion abduction external rotation test, FBC full blood count, NSAIDs non-steroid anti-inflammatory drugs, $R W$ review, $S R$ senior registrar, $T \& O$ trauma and orthopaedics
Pathway for Conservative Management of Low Energy (fragility) Pelvic Fractures in Older Adults ( $\geq 65$ years).

Older Adult $(\geq 65 \mathrm{yrs})$ admitted to Emergency Department (CDU) or care of the elderly wards Pelvic Fracture/s suspected or diagnosed

П.

Please make sure they have available the following:

+ Haemodynamic \& Clotting \& Neurovascular Assessment

+ Falls Risk Assessment

+ AP pelvis

+ Clinical examination of post pelvis - (palpation FABER Sacral compression Gaenslen tests) $+/$-CT pelvis (or Inlet / Outlet views if CT pelvis not feasible) then D/W T\&O on-call bleep holder

$$
\checkmark
$$

T\&O on-call bleep holder will discuss case with on-call Senior T\&O Medical Staff (SR / Consultant) Case will be inserted in Discussion Section of the Daily Trauma List Case will be presented on the next Trauma Meeting

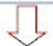

If Consensus Plan is for conservative management / no surgery and then is not for admission to $T \& O$ ward follow the CONSERVATIVE MANAGEMENT PLAN on the next page: were under any of these agents or started those within the first six weeks from their trauma, versus those that did not receive any therapy for osteoporosis.

Pre- and post-injury mobilization status was categorized into four categories: 1: unaided community ambulatory, 2: aided community ambulatory, 3: household ambulatory and 4: non-functional ambulatory.

Radiological healing was assessed via standard anteroposterior, inlet and outlet X-rays and was defined as the presence of bridging callus. Malunion was defined as sagittal malrotation or vertical displacement of the hemipelvis of $\geq 15$ degrees or $\geq 10 \mathrm{~mm}$ respectively, or a displacement/step between united fragments of $\geq 10 \mathrm{~mm}$ [15].

Clinical healing was recorded when a physician or attending physiotherapist recorded pain-free function of the patient and/or return to pre-injury level of mobility at the outpatient follow-up appointment or outpatient physiotherapy session.

The pre- and post-injury mobility status was scored in two ways: by category and by absolute return to pre-injury mobility (recorded by the attending physiotherapist, or clinician at follow-up appointments).

The primary endpoint of the study was the time to healing as recorded either clinically and/or radiologically (whichever was recorded first). Secondary endpoints were considered the length of hospital stay (LOS), time to discharge from followup, complications (including non-union and malunion of the pelvic ring), mortality, return to pre-injury mobility status and return to pre-injury residence.

Descriptive statistics were used for patient characteristics. For the statistical analysis, we used $R$ : A language and environment for statistical computing. To adjust for confounders, comparison was done through modelling and consequent adjustments. For dichotomous outcomes, multivariable logistic regression analysis was employed to identify patient characteristics associated with return to pre-injury mobility, complications, and malunion. A $p$-value of $10 \%$ was used as cut-off point, as standard errors were expected to be larger in a relatively small data set as the presented one. After the initial analysis, the multivariable logistic regression analysis was refitted with only the selected potential risk factors to create a more parsimonious model so that precision of the estimates was increased. Odds ratios were reported for comparison.

Fracture healing as an outcome was classified as nonunion, partial union or malunion, or complete union and consequently an ordered logistic regression was employed. For LOS regression, analysis was undertaken following a logarithm transformation to achieve variance stability. Variables with a T-value of $-1.5>T<1.5$ were eliminated from the model. Time-to-healing was modelled with survival models: initially with Kaplan-Maier survival curves and then with Cox proportional hazards models. To estimate the value of each variable the Wald test was used. Interrater reliability for nonunion, union, and malunion was calculated using weighted kappa-values, and Cohen's kappa-values were used. $P$-values of $<0.05$ (2-sided) were considered as statistically significant.

\section{Results}

During the above pre-specified period, out of 209 consecutive patients managed in our institution, 132 met the inclusion criteria (Fig. 3; flowchart of selection criteria). The included 


\section{CONSERVATIVE MANAGEMENT PLAN PELVIC FRACTURES + 65yrs

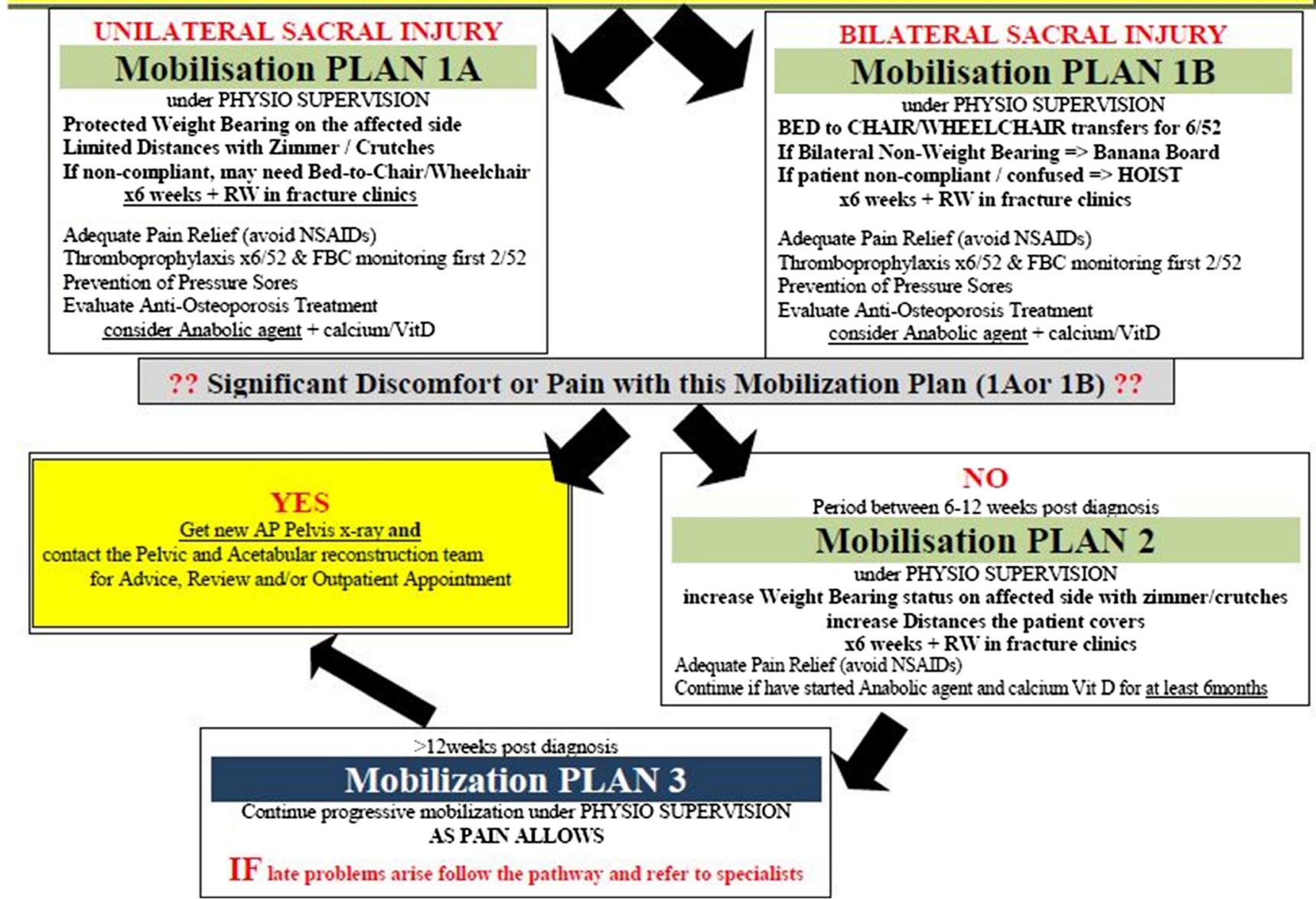

Fig. 2 The algorithm of non-operative management (FPF-SOPstandard operating procedure) of low energy fractures of the pelvic ring that was tested in this study. $A P$ anteroposterior X-ray, $C D U$ clinical decision unit, $C T$ computed tomography, $D / W$ discuss with, FABER test flexion abduction external rotation test, $F B C$ full blood count, NSAIDs non-steroid anti-inflammatory drugs, $R W$ review, $S R$ senior registrar, $T \& O$ trauma and orthopaedics patients consisted of 108 females (108/132; 81.8\%) and 24 males, with a mean age of 85.8 years (SD 7.9; range 67108). A total of 130 patients $(130 / 132 ; 98.5 \%)$ underwent plain X-rays; 113 cases $(113 / 132 ; 85.6 \%)$ had a CT-scan, and 11 patients $(11 / 132 ; 8.3 \%)$ had a pelvic MRI scan. Anterior pelvic lesions were documented in 124 patients $(124 / 132 ; 93.9 \%)$, whereas posterior lesions were noted in 119 cases $(119 / 132 ; 90.2 \%)$.

Of the 121 patients with a superior pubic rami fracture, 102 had also a posterior fracture $(102 / 121 ; 84.3 \%)$. In patients with a unilateral anterior fracture, the posterior injury was found on the ipsilateral side in $75.6 \%$ of the cases, in $8.9 \%$ on the contralateral side and $15.6 \%$ showed a bilateral posterior fracture pattern. Patients with a bilateral anterior fracture showed an even distribution of right side, left side and bilateral posterior fractures. LOS was notoriously varied, with a mean of 22.4 days (SD 19.8) and median 17 days (range 1-106). Thirty-six patients $(27.3 \%)$ died within the 12-month follow- up period, of whom $12(9 \%)$ died without leaving the hospital following their FPF admission. Analysis for hospital LOS was restricted to patients that were discharged alive $(120,91 \%)$. Of 132 patients, 43 (43/132;32.6\%) had a recorded complication. Infections were reported in four patients $(4 / 132 ; 3.0 \%)$ : one urinary tract infection and three respiratory tract infections. Two developed a DVT, and one a pulmonary embolism. A subsequent fall was reported in 13 patients $(13 / 132 ; 9.8 \%)$ and six patients had surgery due to new injuries following the second fall. Eleven $(11 / 132 ; 8.3 \%)$ patients died within six months.

Out of the survivors with complete data on their pre-/postinjury mobilization data, 60 patients $(56.6 \%)$ regained their pre-injury mobility state, 34 (32\%) lost a single level, 12 (11.3\%) fell back by two levels, whilst 12 had incomplete observations and 14 died prematurely (Fig. 4). There were no patients with a better mobility score post-injury than preinjury. 
PELVIC RING fractures of Elderly patients

referred and/or admitted to Trauma and Orthopaedics between January 2010 and December 2014

$\mathrm{N}=\mathbf{2 0 9}$

LOW ENERGY TRAUMA

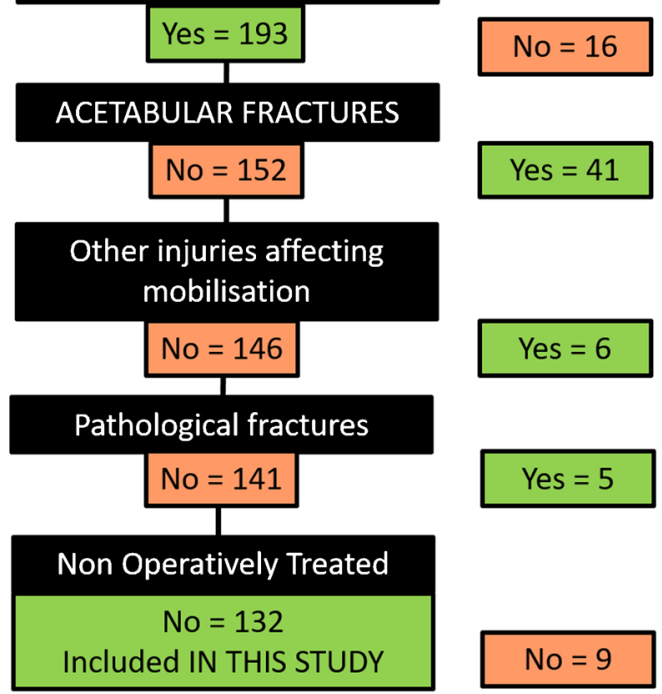

Fig. 3 Flowchart of selecting the study cohort of pelvic ring fractures of patients above 65 years of age that were referred to the department over the study period 2010-2012

The FPF-SOP protocol (group B) was applied in 67 patients $(67 / 132 ; 50.8 \%)$, with the remaining $65(65 / 132$; $49.2 \%$ ) managed without following the protocol, as this was introduced a year later (group A). More detailed patient characteristics of the groups categorized as to their adherence to the FPF-SOP are described at Table 1.

During the first six weeks, 87 patients $(87 / 132 ; 65.9 \%)$ were on anti-osteoporotic medication. The basic characteristics of this cohort of patients and their outcomes according to the use or not of antiosteoporotic drugs are presented at Table 2.

A model fitting age, gender, following the FPF-SOP protocol, using anti-osteoporotic medication and sacral fracture severity was created for regaining absolute pre-injury mobility. It was found that male gender (OR 5.57), Charlson comorbidity index (OR 1.24) and following the FPF-SOP protocol (OR 2.54) were significantly associated with regaining prior mobility (Table 3 ).

Men, with less unstable pelvic fracture, and unaided community pre-injury mobility appeared to be admitted for a period shorter than the median stay of 17 days, in contrast to female patients, with more unstable pelvic fractures and problematic pre-injury mobility. However, the logistic model fitted for age, gender, following the FPF-SOP protocol, using antiosteoporotic medication and sacral fracture severity identified no significant associations. There was little evidence of association for developing a complication or with the LOS with age, gender, Charlson comorbidity index, using anti-osteoporotic drug or following the FPF-SOP protocol (Table 3). A more severe posterior injury (Hannover type $\geq 2$ ) showed more correlation, however was not statistically significant.

To assess final radiological outcome a multivariate model was fitted including age, male gender, Charlson index, following the FPF-SOP protocol and use of anti-osteoporotics. Analysing factors associated with FPF healing, one at a time, we identified that there was no difference in healing time by age group, Charlson group or Hannover classification (Table 3). Patients following the FPF-SOP protocol did not demonstrate a shorter time to healing, whilst an association was found for male gender with time of healing, though not significant ( $p$-value 0.063 ).

The use of anti-osteoporotics (Fig. 5) seemed to have a significant positive effect also ( $p$-value 0.036 ), though we could not distinguish between types of antiosteoporotics. Fitting a Cox regression model, there was evidence for faster healing for those prescribed anti-osteoporotic medication $(p$ value 0.036 ).

It was noted that the adherence to the FPF-SOP protocol led to a better radiological outcome (less malunions; $p$-value 0.031). Weighted kappa for healing showed agreement of 0.77. A survival approach was used for the analysis of time to healing, with survival probability translated to healing probability.
Fig. 4 Patients' mobility score as recorded reflecting their pre-injury mobility status and one recorded at final follow up

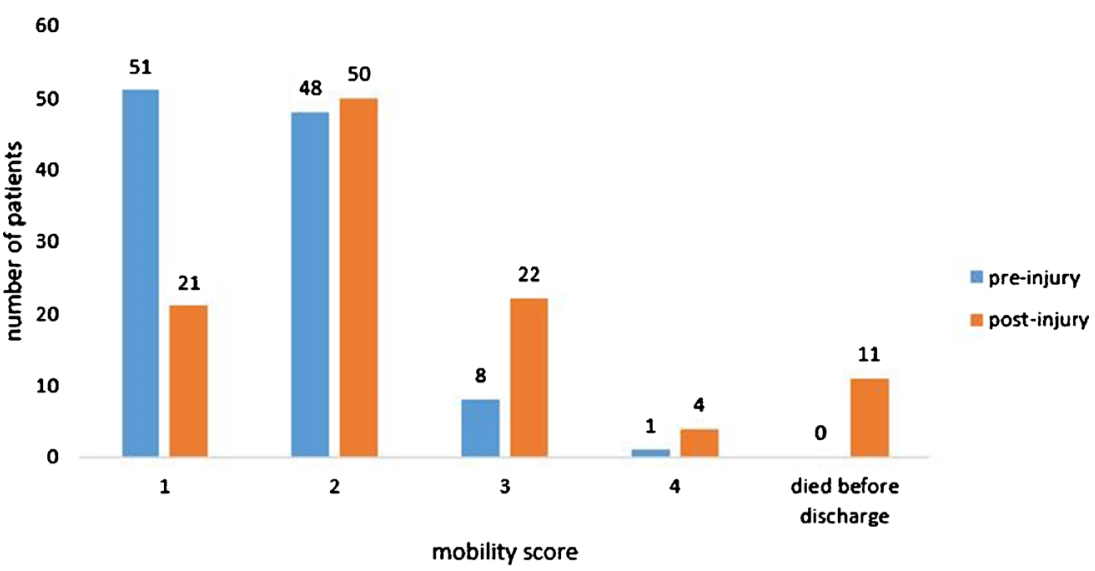


Table 1 Basic characteristics of the patients and their outcome stratified as to the adherence or not tot the FPF SOP

\begin{tabular}{|c|c|c|}
\hline & $\begin{array}{l}\text { Group A following } \\
\text { the SOP }\end{array}$ & $\begin{array}{l}\text { Group B Not following } \\
\text { the SOP }\end{array}$ \\
\hline \multicolumn{3}{|l|}{ General characteristics } \\
\hline Number, $\%$ & $67,50.8 \%$ & $65,49.2 \%$ \\
\hline Males & $13,19.4 \%$ & $11,16.9 \%$ \\
\hline Females & $54,80.6 \%$ & $54,83.1 \%$ \\
\hline Age years mean, $(\mathrm{SD})$ & $86,(8.1)$ & $84.2,(6.7)$ \\
\hline Charlson comorbidity index median, (range) & $5.6,(2.4)$ & $5.5,(2.5)$ \\
\hline \multicolumn{3}{|l|}{ Type of drugs } \\
\hline Vitamin D and calcium & $54,80.6 \%$ & $30,46.2 \%$ \\
\hline Bisphosphonates & $36,53.7 \%$ & $20,30.8 \%$ \\
\hline Strontium & $4,6 \%$ & $5,7.7 \%$ \\
\hline DEXA scan prior to FPF & $27,38.7 \%$ & $16,24.6 \%$ \\
\hline Known osteoporosis & $20,29.9 \%$ & $13,20 \%$ \\
\hline \multicolumn{3}{|l|}{ Diagnosis of the FPFs } \\
\hline With plain $\mathrm{x}$-rays & $66,98.5 \%$ & $64,98.5 \%$ \\
\hline With CT scan & $57,85.1 \%$ & $56,86.2 \%$ \\
\hline With MRI scan & $6,9 \%$ & $5,7.7 \%$ \\
\hline Anterior fractures & $60,91 \%$ & $61,93.8 \%$ \\
\hline Nakatani 1 & $6,9 \%$ & $15,23.1 \%$ \\
\hline Nakatani 2 & $29,43.3 \%$ & $14,21.5 \%$ \\
\hline Nakatani 3 & $25,38.8 \%$ & $32,49.2 \%$ \\
\hline Posterior lesions & $56,83.6 \%$ & $49,75.4 \%$ \\
\hline Hannover type $1 \mathrm{a}, 1 \mathrm{~b}$ & $42,77.6 \%$ & $29,44.6 \%$ \\
\hline Hannover type $2 \mathrm{a}, 2 \mathrm{~b}, 3,4$ & $12,17.9 \%$ & $20,30.8 \%$ \\
\hline Iliac Blade fracture & $2,3 \%$ & $6,9.2 \%$ \\
\hline \multicolumn{3}{|l|}{ Pre-injury mobilization status } \\
\hline Unaided community ambulatory & $28,41.8 \%$ & $26,40 \%$ \\
\hline Aided community ambulatory & $24,35.8 \%$ & $27,41.5 \%$ \\
\hline Household ambulatory & $5,7.5 \%$ & $4,6.1 \%$ \\
\hline Non-functional ambulatory & $0,0 \%$ & $0,0 \%$ \\
\hline Non-available data & $10,14.9 \%$ & $8,12.3 \%$ \\
\hline \multicolumn{3}{|l|}{ Post-injury at final follow up mobilization status } \\
\hline Unaided community ambulatory & $12,17.9 \%$ & $9,13.8 \%$ \\
\hline Aided community ambulatory & $32,47.8 \%$ & $19,29.2 \%$ \\
\hline Household ambulatory & $10,14.9 \%$ & $15,23.1 \%$ \\
\hline Non-functional ambulatory & $0,0 \%$ & $4,6.2 \%$ \\
\hline Died earlier & $8,11.9 \%$ & $4,6.2 \%$ \\
\hline Non-available data & $5,7.7 \%$ & $14,21.5 \%$ \\
\hline \multicolumn{3}{|l|}{ Outcome } \\
\hline LOS in days mean, (SD) & $21.5,(19)$ & $21,(18.5)$ \\
\hline Union & $43,64.2 \%$ & $27,41.5 \%$ \\
\hline Malunion & $4,6 \%$ & $17,26.2 \%$ \\
\hline Nonunion & $2,3 \%$ & $2,3.1 \%$ \\
\hline Time to radiological healing mean, $(\mathrm{SD})$ & 168 days $(229.7)$ & 166.2 days $(231.5)$ \\
\hline Time to clinical healing mean, (SD) & 139.8 days $(116.6)$ & 138.1 days (117.4) \\
\hline Complications & $25,37.3 \%$ & $26,40 \%$ \\
\hline Death in-hospital within the same admission & $3,4.5 \%$ & $9,13.8 \%$ \\
\hline Death within 12 months from FPF & $18,26.9 \%$ & $26,40 \%$ \\
\hline Return to pre-injury residence & $35,52.2 \%$ & $46,70.8 \%$ \\
\hline Restoration of the pre-injury mobility status & $38,56.7 \%$ & $22,33.8 \%$ \\
\hline
\end{tabular}


Table 1 (continued)

\begin{tabular}{lll}
\hline & $\begin{array}{l}\text { Group A following } \\
\text { the SOP }\end{array}$ & $\begin{array}{l}\text { Group B Not following } \\
\text { the SOP }\end{array}$ \\
\hline Loss of one level of the pre-injury mobility status & $17,25.4 \%$ & $17,26.2 \%$ \\
Loss of two levels of the pre-injury mobility status & $5,7.5 \%$ & $7,10.8 \%$ \\
\hline
\end{tabular}

$D E X A$ dual-energy x-ray absorptiometry, $F P F$ fragility pelvic fracture, $m$ months, $L O S$ length of stay, $S D$ standard deviation, $S O P$ standard operating protocol

\section{Discussion}

The objective of this study was to determine the effect of using a specific management protocol and the use of antiosteoporotic medication on fracture healing in low energy pelvic fractures in the elderly population.

It is our belief that the main goal of treatment for the FPFs should be similar to that of hip fractures: focusing on the optimisation of medical comorbidities, early ambulation, and early hospital discharge to an appropriate facility. A significant difference to the hip fractures is the fact that the FPFs mainstay of treatment is non-operative. This approach is due to the following: (a) a significant proportion of these fractures are thought to be mechanically stable; (b) limitations of current pelvic fixation techniques in the presence of severe osteoporosis; (c) the high perioperative morbidity of open pelvic surgery in this frail cohort of patients; and finally, (d) the relatively scarce availability of trained pelvic trauma surgeons. Nevertheless, there are specific indications for surgical treatment of FPFs, especially of the posterior elements, usually with minimal invasive or percutaneous techniques [16-18].

It is of interest that inadequate initial management in combination with the poor bone quality and the slow progress of fracture healing [19] could lead to further displacement of bony fragments, development of additional fracture lines, secondary loss of reduction, implant failure, non-union and poor clinical outcome [18]. Initial imaging in these patients with a suspected low energy pelvic ring injury is usually limited to plain X-rays. However, although plain X-rays are considered an adequate tool for diagnosis of anterior ring pathologies, their accuracy in the evaluation of posterior pelvic ring lesions is limited, due to the low bone density, the interposing viscera $[6,16]$, and the minimal initial displacement of FPFs. As a result sacral insufficiency fractures are often overlooked in plain X-rays of patients presenting with low back and/or pelvic pain [20]. Fractures of the pubis, which are visible on the X-ray, are nearly always associated with injuries of the posterior pelvic elements [21]. As most forces are transmitted through the posterior pelvic ring and the posterior joints [22], posterior fractures are viewed as more crucial than anterior fractures, and some clinicians and researchers plead for standardizing the use of CT scans in the diagnosis of elderly people presenting with low energy pelvic injury [17]. As shown also to our cohort, balancing decision making on plain imaging would mislead the mobilization guidance in $24.4 \%$. Having objective evidence of the posterior pelvic fractures will certainly have an important part in the positive effect of the protocol, as this allows for a more effective management in protecting the affected posterior side in each case. All the above supports our decision to include an early acquisition of a pelvic $\mathrm{CT}$ scan in the SOP.

In this study, some shortcomings should be considered. As in every cohort study not all the data were available for every patient. Also, randomized matched groups could not be evaluated. With regard to the time of healing, as in all fracture healing studies, the follow-up is not continuous. Follow-up imaging and out-patient visits are grossly dependent on logistics of outpatient departments. In a structured prospective study with set times of follow-up one might explore these outcomes better.

In general, it is hard to get absolute agreement regarding fracture healing assessment among clinicians in most clinical studies. Besides that, the majority of studies do not specify their definition of healing, or even indicate who functioned as the observer [23]. In contrast, we attempted to improve by assigning two blinded independent observers, and our data analysis demonstrated good consensus.

The concept of acceleration of fracture healing in this group of patients has attracted significant scientific attention [19, 24]. Consequently, the strategy of using anti-osteoporotic drugs in the medical treatment of FPFs represents an exciting prospect $[14,25]$. We were not able to distinguish this effect between different groups of medications, although the literature suggests a clear positive effect on fracture healing of anabolic type medication [26]. Only a few patients using anti-osteoporotic medication $(N=9 ; 10.3 \%)$ were using strontium ranelate, and no use of teriparatide was documented. This was against expectations, as within the FPF-SOP there was clear advice to the patients' orthogeriatricians/general practitioners to start anabolic agents. Presumably this reflects the relative high costs of these drugs, as well as the adherence of primary care physicians to the existing therapeutic guidelines [27].

However, this study showed that the use of anti-osteoporotic medication in general, shortens the time to healing significantly ( $p=0.036)$, confirming our hypothesis. Most patients in the study that were receiving anti-osteoporotic therapy used bisphosphonates $(N=57 ; 65.5 \%)$, reflecting the existing mainstream therapeutic protocols in the United Kingdom [27]. In 
Table 2 Basic characteristics of the patients and their outcome stratified as to the use or not of antiosteoporotic agents

\begin{tabular}{|c|c|c|c|}
\hline & $\begin{array}{l}\text { Patients group receiving } \\
\text { antiosteoporotic drugs }\end{array}$ & $\begin{array}{l}\text { Patients group not receiving } \\
\text { antiosteoporotic drugs }\end{array}$ & $\begin{array}{l}\text { Patients group with } \\
\text { no available data }\end{array}$ \\
\hline \multicolumn{4}{|l|}{ General characteristics } \\
\hline Number, $\%$ & $87,65.9 \%$ & $19,14.4 \%$ & $26,19.7 \%$ \\
\hline Males & $13,14.9 \%$ & $3,15.8 \%$ & $8,30.7 \%$ \\
\hline Females & $74,85.1 \%$ & $16,84.2 \%$ & $18,69.2 \%$ \\
\hline AGE years mean, (SD) & $85.3,(7.6)$ & $83.5,(6.6)$ & $85.6,(8.0)$ \\
\hline Charlson comorbidity index median, (range) & $5.6(2.3)$ & $5.4,(1.3)$ & $5.7,(3.2)$ \\
\hline \multicolumn{4}{|l|}{ Type of drugs } \\
\hline Vitamin D and calcium & $84,96.6 \%$ & $\mathrm{n} / \mathrm{a}$ & $\mathrm{n} / \mathrm{a}$ \\
\hline Bisphosphonates & $56,65.5 \%$ & $\mathrm{n} / \mathrm{a}$ & $\mathrm{n} / \mathrm{a}$ \\
\hline Strontium & $9,10.3 \%$ & $\mathrm{n} / \mathrm{a}$ & $\mathrm{n} / \mathrm{a}$ \\
\hline DEXA scan prior to FPF & $33,37.9 \%$ & $7,36.8 \%$ & $2,7.7 \%$ \\
\hline Known osteoporosis & $25,28.7 \%$ & $6,31.6 \%$ & $2,7.7 \%$ \\
\hline \multicolumn{4}{|l|}{ Diagnosis of the FPFs } \\
\hline With plain x-rays & $87,100 \%$ & $18,94.7 \%$ & $25,96.1 \%$ \\
\hline With CT scan & $77,88.5 \%$ & $17,89.5 \%$ & $19,73.1 \%$ \\
\hline With MRI scan & $4,4.6 \%$ & $3,15.8 \%$ & $4,15.4 \%$ \\
\hline Anterior fractures & $82,94.3 \%$ & $18,94.7 \%$ & $23,88.5 \%$ \\
\hline Posterior lesions & $73,83.9 \%$ & $18,94.7 \%$ & $20,76.9 \%$ \\
\hline \multicolumn{4}{|l|}{ Pre-injury mobilization status } \\
\hline Unaided community ambulatory & $37,42.5 \%$ & $6,31.6 \%$ & $10,38.5 \%$ \\
\hline Aided community ambulatory & $32,36.8 \%$ & $9,47.4 \%$ & $11,42.3 \%$ \\
\hline Household ambulatory & $7,8 \%$ & $5.3 \%$ & $1,3.8 \%$ \\
\hline Non-functional ambulatory & $0,0 \%$ & $0,0 \%$ & $0,0 \%$ \\
\hline Non-available data & $11,12.6 \%$ & $3,15.8 \%$ & $4,15.4 \%$ \\
\hline \multicolumn{4}{|l|}{ Post-injury at final follow up mobilization status } \\
\hline Unaided community ambulatory & $15,17.2 \%$ & $0,0 \%$ & $6,23.1 \%$ \\
\hline Aided community ambulatory & $35,40.2 \%$ & $14,73.7 \%$ & $2,7.7 \%$ \\
\hline Household ambulatory & $22,25.3 \%$ & $1,5.3 \%$ & $3,11.5 \%$ \\
\hline Non-functional ambulatory & $0,0 \%$ & $1,5.3 \%$ & $2,7.7 \%$ \\
\hline Died earlier & $4,4.6 \%$ & $1,5.3 \%$ & $7,26.9 \%$ \\
\hline Non-available data & $11,12.6 \%$ & $2,10.5 \%$ & $6,23.1 \%$ \\
\hline \multicolumn{4}{|l|}{ Outcome } \\
\hline LOS mean, (SD) & $21.5,(17.4)$ & $24.3,(19.6)$ & $21.5,(22.5)$ \\
\hline Union & $53,60.9 \%$ & $11,57.9 \%$ & $6,23.1 \%$ \\
\hline Malunion & $9,10.3 \%$ & $7,36.8 \%$ & $5,19.2 \%$ \\
\hline Nonunion & $2,2.3 \%$ & $2,10.5 \%$ & $0,0 \%$ \\
\hline Time to radiological healing mean, (SD) & $169.6,(230.5)$ & $177.8,(240.2)$ & $169.9,(233.2)$ \\
\hline Time to clinical healing mean, (SD) & $140.9,(116.6)$ & $144.9,122.9)$ & $136.7,(114.1)$ \\
\hline Complications & $29,33.3 \%$ & $5,26.3 \%$ & $17,65.4 \%$ \\
\hline Death in-hospital within the same admission & $0,0 \%$ & $0,0 \%$ & $12,46.2 \%$ \\
\hline Death within $12 \mathrm{~m}$ from FPF & $20,23 \%$ & $3,15.8 \%$ & $21,80.8 \%$ \\
\hline Return to pre-injury residence & $55,63.2 \%$ & $14,73.7 \%$ & $12,46.2 \%$ \\
\hline Restoration of the pre-injury mobility status & $45,51.7 \%$ & $8,42.1 \%$ & $7,26.9 \%$ \\
\hline Loss of one level of the pre-injury mobility status & $24,27.6 \%$ & $7,36.8 \%$ & $3,11.5 \%$ \\
\hline Loss of two levels of the pre-injury mobility status & $8,9.2 \%$ & $2,10.5 \%$ & $2,7.7 \%$ \\
\hline
\end{tabular}

$D E X A$ dual-energy x-ray absorptiometry, $F P F$ fragility pelvic fracture, $m$ months, $L O S$ length of stay, $S D$ standard deviation, $S O P$ standard operating protocol 
Table 3 Effect to the secondary outcome measures of associated risk factors as identified and weighted following logistic regression analysis

\begin{tabular}{|c|c|c|c|c|}
\hline \multirow[t]{3}{*}{ Risk factors } & \multicolumn{4}{|c|}{ Secondary outcomes } \\
\hline & \multirow{2}{*}{$\begin{array}{l}\text { First model } \\
P \text {-value }\end{array}$} & \multicolumn{3}{|c|}{ Corrected model } \\
\hline & & Odds ratio & 95\% Confidence interval $(\mathrm{CI})$ & $P$-value \\
\hline \multicolumn{5}{|l|}{ Length of stay $(\mathrm{LOS})^{\mathrm{a}}$} \\
\hline Age & 0.46 & $\mathrm{n} / \mathrm{a}$ & $\mathrm{n} / \mathrm{a}$ & $\mathrm{n} / \mathrm{a}$ \\
\hline Gender (male) & 0.30 & $\mathrm{n} / \mathrm{a}$ & $\mathrm{n} / \mathrm{a}$ & $\mathrm{n} / \mathrm{a}$ \\
\hline Charlson comorbidity index & 0.61 & $\mathrm{n} / \mathrm{a}$ & $\mathrm{n} / \mathrm{a}$ & $\mathrm{n} / \mathrm{a}$ \\
\hline Following the SOP & 0.29 & $\mathrm{n} / \mathrm{a}$ & $\mathrm{n} / \mathrm{a}$ & $\mathrm{n} / \mathrm{a}$ \\
\hline On anti-osteoporotic drugs & 0.60 & $\mathrm{n} / \mathrm{a}$ & $\mathrm{n} / \mathrm{a}$ & $\mathrm{n} / \mathrm{a}$ \\
\hline Hannover $>1$ & 0.54 & $\mathrm{n} / \mathrm{a}$ & $\mathrm{n} / \mathrm{a}$ & $\mathrm{n} / \mathrm{a}$ \\
\hline \multicolumn{5}{|l|}{ Complications $^{\mathrm{b}}$} \\
\hline Age & 0.69 & $\mathrm{n} / \mathrm{a}$ & $\mathrm{n} / \mathrm{a}$ & $\mathrm{n} / \mathrm{a}$ \\
\hline Gender (male) & 0.22 & $\mathrm{n} / \mathrm{a}$ & $\mathrm{n} / \mathrm{a}$ & $\mathrm{n} / \mathrm{a}$ \\
\hline Charlson index & 0.89 & $\mathrm{n} / \mathrm{a}$ & $\mathrm{n} / \mathrm{a}$ & $\mathrm{n} / \mathrm{a}$ \\
\hline Following the SOP & 0.60 & $\mathrm{n} / \mathrm{a}$ & $\mathrm{n} / \mathrm{a}$ & $\mathrm{n} / \mathrm{a}$ \\
\hline On anti-osteoporotic drugs & 0.46 & $\mathrm{n} / \mathrm{a}$ & $\mathrm{n} / \mathrm{a}$ & $\mathrm{n} / \mathrm{a}$ \\
\hline Hannover $>1$ & 0.17 & $\mathrm{n} / \mathrm{a}$ & $\mathrm{n} / \mathrm{a}$ & $\mathrm{n} / \mathrm{a}$ \\
\hline \multicolumn{5}{|l|}{ Malunion $^{\mathrm{b}}$} \\
\hline Age & 0.8785 & $\mathrm{n} / \mathrm{a}$ & $\mathrm{n} / \mathrm{a}$ & $\mathrm{n} / \mathrm{a}$ \\
\hline Gender (male) & 0.6951 & $\mathrm{n} / \mathrm{a}$ & $\mathrm{n} / \mathrm{a}$ & $\mathrm{n} / \mathrm{a}$ \\
\hline Charlson index & 0.4964 & $\mathrm{n} / \mathrm{a}$ & $\mathrm{n} / \mathrm{a}$ & $\mathrm{n} / \mathrm{a}$ \\
\hline Following the SOP & 0.0036 & 0.13 & $0.03-0.38$ & 0.0006 \\
\hline On anti-osteoporotic drugs & 0.4246 & $\mathrm{n} / \mathrm{a}$ & $\mathrm{n} / \mathrm{a}$ & $\mathrm{n} / \mathrm{a}$ \\
\hline Hannover $>1$ & 0.5259 & $\mathrm{n} / \mathrm{a}$ & $\mathrm{n} / \mathrm{a}$ & $\mathrm{n} / \mathrm{a}$ \\
\hline \multicolumn{5}{|l|}{ Time to healing ${ }^{\mathrm{c}}$} \\
\hline Age & 0.786 & $\mathrm{n} / \mathrm{a}$ & $\mathrm{n} / \mathrm{a}$ & $\mathrm{n} / \mathrm{a}$ \\
\hline Gender (male) & 0.093 & 1.71 & $0.97-3.02$ & 0.063 \\
\hline Charlson index & 0.480 & $\mathrm{n} / \mathrm{a}$ & $\mathrm{n} / \mathrm{a}$ & $\mathrm{n} / \mathrm{a}$ \\
\hline Following the SOP & 0.464 & $\mathrm{n} / \mathrm{a}$ & $\mathrm{n} / \mathrm{a}$ & $\mathrm{n} / \mathrm{a}$ \\
\hline On anti-osteoporotic drugs & 0.023 & 1.79 & $1.04-3.07$ & 0.036 \\
\hline \multicolumn{5}{|l|}{ Nonunion $^{\mathrm{d}}$} \\
\hline Age & -0.224 & $\mathrm{n} / \mathrm{a}$ & $\mathrm{n} / \mathrm{a}$ & $\mathrm{n} / \mathrm{a}$ \\
\hline Gender (male) & 0.554 & $\mathrm{n} / \mathrm{a}$ & $\mathrm{n} / \mathrm{a}$ & $\mathrm{n} / \mathrm{a}$ \\
\hline Charlson index & 0.933 & $\mathrm{n} / \mathrm{a}$ & $\mathrm{n} / \mathrm{a}$ & $\mathrm{n} / \mathrm{a}$ \\
\hline Following the SOP & 1.731 & 2.19 & 0.031 & $\mathrm{n} / \mathrm{a}$ \\
\hline On anti-osteoporotic drugs & 1.183 & $\mathrm{n} / \mathrm{a}$ & $\mathrm{n} / \mathrm{a}$ & $\mathrm{n} / \mathrm{a}$ \\
\hline \multicolumn{5}{|c|}{ Return to pre-injury mobility status ${ }^{\mathrm{b}}$} \\
\hline Age & 0.2004 & $\mathrm{n} / \mathrm{a}$ & $\mathrm{n} / \mathrm{a}$ & $\mathrm{n} / \mathrm{a}$ \\
\hline Gender (male) & 0.0057 & 5.57 & $1.88-18.39$ & 0.0028 \\
\hline Charlson index & 0.0336 & 1.24 & $1.02-1.55$ & 0.0383 \\
\hline Following the SOP & 0.0378 & 2.54 & $1.07-6.35$ & 0.0389 \\
\hline On anti-osteoporotic drugs & 0.6740 & $\mathrm{n} / \mathrm{a}$ & $\mathrm{n} / \mathrm{a}$ & $\mathrm{n} / \mathrm{a}$ \\
\hline Hannover $>1$ & 0.4784 & $\mathrm{n} / \mathrm{a}$ & $\mathrm{n} / \mathrm{a}$ & $\mathrm{n} / \mathrm{a}$ \\
\hline
\end{tabular}

In Bold: statistically significant results

$L O S$ length of stay, $n / a$ not applicable, $S O P$ standard operating procedure

Analysis restricted to those discharged alive

${ }^{a}$ Regression analysis with log transformation ${ }^{\mathrm{b}}$ Logistic regression analysis

${ }^{\mathrm{c}}$ Cox regression model ${ }^{\mathrm{d}}$ Polytomous linear regression analysis 
Fig. 5 Graphic representation of the positive effect of the use of antiosteoporotic agents to the recorded time to clinical/ radiological healing; Kaplan Meier curve

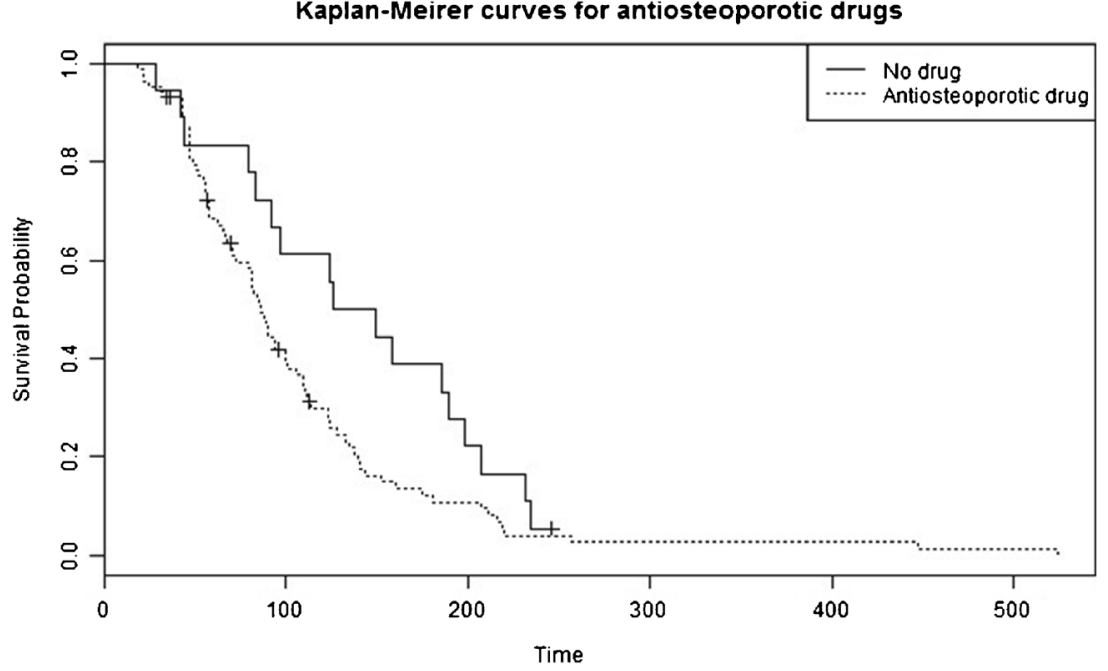

summary, calcium and vitamin D and generic alendronate (bisphosphonate) are considered as the first line of treatment. If alendronate is contraindicated, other bisphosphonates, RANKL inhibitors, or strontium ranelate are used. Parathyroid hormone peptides (PTH-teriparatide) are only used in patients at very high risk due to their high cost [28].

The use of bisphosphonates in the presence of a fracture is associated with concerns of their interaction with bone remodelling and impaired fracture healing. However, studies have shown larger/stronger callus and delayed remodelling but no evidence of delayed healing [14]. Studies on RANKL inhibitors showed greater callus volume and mineral density, and no delay on fracture healing [29]. Experimental data for strontium ranelate range from no effect to significant increase in both callus volume and strength [14]. PTH has demonstrated accelerated healing in various experimental trials in humans [26, 30]. Peichl et al. [26] showed a significant difference between time to healing in patients with fragility fractures of the pelvis in patients receiving PTH 1-84 compared to the control group. The potential effect on fracture healing of sclerostin blockers is yet to be studied [14].

Our findings underline the theory that the use of bisphosphonates does not impair fracture healing, and, on the contrary, seems to enhance it. However, such findings could be associated with other confounding factors. We suspect that patients on anti-osteoporotics receive better care in general, and are better guided by their environment. Also, bisphosphonates are known to be notoriously difficult to use and are poorly absorbed from the gastrointestinal tract [31], which makes it more likely that the outcome is also affected by other variables.

Following the FPF-SOP protocol appeared to protect against the development of malunion ( $p<0.001)$, an association which is consistent when looking at overall final healing outcome ( $p=0.031$ ). This could be explained as the affected side is more effectively protected due to the early and accurate diagnosis. These patients are supported by physiotherapists all the way, as opposed to having patients mobilized as-pain-allows. Patients following the protocol were also more than twice as likely to regain pre-injury mobility $(\mathrm{OR}=2.54, p=0.039)$. This might be because they have had better guidance throughout the process.

The use of the SOP was not associated with a reduction of the LOS. This can possibly be explained due to the fact that LOS of the elderly in an acute hospital is governed by multiple other factors [4]; in most, by the availability of a bed in nursing homes or short-stay facilities. There might be room for improvement in the cooperation between hospital and nursing homes and perhaps a universal protocol can help with that. The clinical significance of these results is yet to be assessed.

An unexpected result was the significant association between male gender and regaining mobility $(p=0.003)$. Males also seem to heal faster, although this finding did not reach statistical significance $(p=0.063)$. This association stands after correction for confounders such as age and comorbidities. To our knowledge there are no previous studies that show an association between male gender and a better outcome after a FPF. The effect of hormonal metabolism, bone density, and the range of disabilities may differ between the two genders of the same age [32] should be further explored as contributors to the recovery of FPFs.

\section{Conclusions}

In conclusion, we observed that the use of anti-osteoporotic medication and the introduction of a more structured management in the form of a protocol have a positive effect on patient outcome in elderly patients with a fragility fracture of the pelvis. Using anti-osteoporotic drugs reduces the time of healing and following the protocol protects against malunion and gives a better chance of regaining pre-injury mobility. 
Acknowledgements We wish to acknowledge the important contribution of Mr. Theo Tosounidis, Dr. Leonidas Mitrogiannis, Mr. Suribabu Gudipati, and Dr. Jack Henry Gilmore to the acquisition of information relevant to this study data.

Authors contributions NKK conceived the study as well as its design, collection of data, preparation of the draft and review of the final manuscript.

TG was involved to the study design, collection of data, analysis, and preparation of the draft.

RMW participated in the design of the study and performed the statistical analysis.

ABVV was involved to the study design, and analysis of data.

PVG was involved in the study design, collection of data, and review of the final manuscript.

\section{Compliance with ethical standards}

Conflict of interest The authors declare that they have no conflict of interest.

Funding There is no funding source.

Ethical approval In accordance with the NHS act of 2006, section 251, the study was carried out with the required institutional board approval under reference number 10444

Informed consent Informed consent was obtained from all individual participants included in the study.

Open Access This article is distributed under the terms of the Creative Commons Attribution 4.0 International License (http:// creativecommons.org/licenses/by/4.0/), which permits unrestricted use, distribution, and reproduction in any medium, provided you give appropriate credit to the original author(s) and the source, provide a link to the Creative Commons license, and indicate if changes were made.

\section{References}

1. Giannoudis PV, Grotz MR, Tzioupis C, Dinopoulos H, Wells GE, Bouamra O, Lecky F (2007) Prevalence of pelvic fractures, associated injuries, and mortality: the United Kingdom perspective. J Trauma 63(4):875-883. doi:10.1097/01.ta.0000242259.67486.15

2. Breuil V, Roux CH, Testa J, Albert C, Chassang M, Brocq O, Euller-Ziegler L (2008) Outcome of osteoporotic pelvic fractures: an underestimated severity. Survey of 60 cases. Joint Bone Spine 75(5):585-588. doi:10.1016/j.jbspin.2008.01.024

3. Balogh Z, King KL, Mackay P, McDougall D, Mackenzie S, Evans JA, Lyons T, Deane SA (2007) The epidemiology of pelvic ring fractures: a population-based study. J Trauma 63(5):1066-1073; discussion 1072-1063. doi:10.1097/TA.0b013e3181589fa4

4. Sullivan MP, Baldwin KD, Donegan DJ, Mehta S, Ahn J (2014) Geriatric fractures about the hip: divergent patterns in the proximal femur, acetabulum, and pelvis. Orthopedics 37(3):151-157. doi:10. 3928/01477447-20140225-50

5. Studer P, Suhm N, Zappe B, Bless N, Jakob M (2013) Pubic rami fractures in the elderly - a neglected injury? Swiss Med Wkly 143: w13859. doi:10.4414/smw.2013.13859

6. Soles GL, Ferguson TA (2012) Fragility fractures of the pelvis. Curr Rev Musculoskelet Med 5(3):222-228. doi:10.1007/s12178-0129128-9
7. Bible JE, Kadakia RJ, Wegner A, Richards JE, Mir HR (2013) Oneyear mortality after isolated pelvic fractures with posterior ring involvement in elderly patients. Orthopedics 36(6):760-764. doi:10. 3928/01477447-20130523-21

8. Alnaib M, Waters S, Shanshal Y, Caplan N, Jones S, St Clair Gibson A, Kader D (2012) Combined pubic rami and sacral osteoporotic fractures: a prospective study. J Orthop Traumatol 13(2): 97-103. doi:10.1007/s10195-012-0182-2

9. Tile M (1984) Fractures of the pelvis and acetabulum. Williams \& Wilkins, Baltimore

10. Burgess AR, Eastridge BJ, Young JW, Ellison TS, Ellison PS Jr, Poka A, Bathon GH, Brumback RJ (1990) Pelvic ring disruptions: effective classification system and treatment protocols. J Trauma 30(7):848-856

11. Denis F, Davis S, Comfort T (1988) Sacral fractures: an important problem. Retrospective analysis of 236 cases. Clin Orthop Relat Res 227:67-81

12. Pohlemann T, Gansslen A, Tscherne H (1992) The problem of the sacrum fracture. Clinical analysis of 377 cases. Orthopade 21(6): 400-412

13. Starr AJ, Nakatani T, Reinert CM, Cederberg K (2008) Superior pubic ramus fractures fixed with percutaneous screws: what predicts fixation failure? J Orthop Trauma 22(2):81-87. doi:10.1097/ BOT.0b013e318162ab6e

14. Larsson S, Fazzalari NL (2012) Anti-osteoporosis therapy and fracture healing. Arch Orthop Trauma Surg 134(2):291-297. doi:10. 1007/s00402-012-1558-8

15. Kanakaris NK, Angoules AG, Nikolaou VS, Kontakis G, Giannoudis PV (2009) Treatment and outcomes of pelvic malunions and nonunions: a systematic review. Clin Orthop Relat Res 467(8):2112-2124. doi:10.1007/s11999-009-0712-2

16. Culemann U, Scola A, Tosounidis G, Pohlemann T, Gebhard F (2010) Concept for treatment of pelvic ring injuries in elderly patients. A challenge. Unfallchirurg 113(4):258-271. doi:10.1007/ s00113-010-1762-3

17. Fuchs T, Rottbeck U, Hofbauer V, Raschke M, Stange R (2011) Pelvic ring fractures in the elderly. Underestimated osteoporotic fracture. Unfallchirurg 114(8):663-670. doi:10.1007/s00113-0112020-z

18. Rommens PM, Wagner D, Hofmann A (2012) Surgical management of osteoporotic pelvic fractures: a new challenge. Eur J Trauma Emerg Surg 38(5):499-509. doi:10.1007/s00068-0120224-8

19. Nikolaou VS, Efstathopoulos N, Kontakis G, Kanakaris NK, Giannoudis PV (2009) The influence of osteoporosis in femoral fracture healing time. Injury 40(6):663-668. doi:10.1016/j.injury. 2008.10.035

20. Tsiridis E, Upadhyay N, Giannoudis PV (2006) Sacral insufficiency fractures: current concepts of management. Osteoporos Int 17(12): 1716-1725. doi:10.1007/s00198-006-0175-1

21. Cosker TD, Ghandour A, Gupta SK, Tayton KJ (2005) Pelvic ramus fractures in the elderly: 50 patients studied with MRI. Acta Orthop 76(4):513-516. doi:10.1080/17453670510044634

22. Leung AS, Gordon LM, Skrinskas T, Szwedowski T, Whyne CM (2009) Effects of bone density alterations on strain patterns in the pelvis: application of a finite element model. Proc Inst Mech Eng $\mathrm{H}$ 223(8):965-979

23. Corrales LA, Morshed S, Bhandari M, Miclau T 3rd (2008) Variability in the assessment of fracture-healing in orthopaedic trauma studies. J Bone Joint Surg Am 90(9):1862-1868. doi:10.2106/ JBJS.G.01580

24. Xu SW, Yu R, Zhao GF, Wang JW (2003) Early period of fracture healing in ovariectomized rats. Chin J Traumatol 6(3):160-166

25. Giannoudis P, Tzioupis C, Almalki T, Buckley R (2007) Fracture healing in osteoporotic fractures: is it really different? A basic 
science perspective. Injury 38(Suppl 1):S90-S99. doi:10.1016/j. injury.2007.02.014

26. Peichl P, Holzer LA, Maier R, Holzer G (2011) Parathyroid hormone 1-84 accelerates fracture-healing in pubic bones of elderly osteoporotic women. J Bone Joint Surg Am 93(17):1583-1587. doi:10.2106/JBJS.J.01379

27. Compston J, Bowring C, Cooper A, Cooper C, Davies C, Francis R, Kanis JA, Marsh D, McCloskey EV, Reid DM, Selby P, National Osteoporosis Guideline G (2013) Diagnosis and management of osteoporosis in postmenopausal women and older men in the UK: National Osteoporosis Guideline Group (NOGG) update 2013. Maturitas 75(4):392-396. doi:10.1016/j.maturitas.2013.05.013

28. NICE Technology Appraisal Guidance 161 (2011) Secondary prevention of osteoporotic fragility fractures in postmenopausal women. National Institute for Health and Care Excellence. http:// pathways.nice.org.uk/pathways/osteoporosis\#content=view- node $\% 3$ Anodes-secondary-prevention-of-osteoporotic-fragility. Accessed May 2015)

29. Adami S, Libanati C, Boonen S, Cummings SR, Ho PR, Wang A, Siris E, Lane J, Group FF-HW, Adachi JD, Bhandari M, de Gregorio L, Gilchrist N, Lyritis G, Moller G, Palacios S, Pavelka K, Heinrich R, Roux C, Uebelhart D (2012) Denosumab treatment in postmenopausal women with osteoporosis does not interfere with fracture-healing: results from the FREEDOM trial. J Bone Joint Surg Am 94(23):2113-2119. doi:10.2106/JBJS.K.00774

30. Aspenberg P, Johansson T (2010) Teriparatide improves early callus formation in distal radial fractures. Acta Orthop 81(2):234-236. doi:10.3109/17453671003761946

31. Lin JH (1996) Bisphosphonates: a review of their pharmacokinetic properties. Bone 18(2):75-85

32. Zhang W, Li S, Feldman MW (2005) Gender differences in activity of daily living of the elderly in rural China: evidence from Chaohu. J Women Aging 17(3):73-89. doi:10.1300/J074v17n03_06 